\title{
Aneurisma do Átrio Direito Associado à Hidropisia Fetal: Diagnóstico por Ecocardiografia Fetal
}

\author{
Right Atrial Aneurysm Associated to Fetal Hydrops: Diagnosis through \\ Fetal Echocardiography
}

\author{
Marcia Ferreira Alves Barberato, Silvio Henrique Barberato, Cláudio Correa Gomes, \\ Sérgio Luis Costa, Alfred Krawiec \\ CEMMEF- Centro de Medicina Materno-Fetal - Curitiba, PR
}

Os aneurismas de átrio direito são entidades raramente relatadas na prática cardiológica, especialmente em vida intra-uterina, e podendo ser confundidos com derrame pericárdico e anomalia de Ebstein da valva tricúspide. Apresentamos revisão da literatura e ilustramos com caso de diagnóstico pré-natal de aneurisma de átrio direito cursando com sinais de hidropisia.
Right atrium aneurysms are entities which are rarely reported in cardiologic practice, especially in intrauterine life, and may be mistaken with pericardial effusion and Ebstein's anomaly. We show a review of the literature and illustrate with a case of prenatal diagnosis of right atrium aneurysm running through with hydropsy signs.
O aneurisma do átrio direito (AD) é uma malformação cardíaca de etiologia desconhecida, raramente encontrada na literatura médica, especialmente quando se trata de diagnóstico pré-natal. Devido à sua raridade, pode ser facilmente confundido com outras cardiopatias que levam à dilatação do $A D$, como a anomalia de Ebstein da valva tricúspide ${ }^{1,2}$. Além disto, sua evolução intra-uterina usualmente silenciosa e o ainda restrito uso da ecocardiografia fetal no rastreamento das cardiopatias congênitas em nosso meio contribuem para que o diagnóstico só seja feito, na maioria das vezes, na idade adulta ${ }^{3,4}$. Apresentamos um caso de diagnóstico intra-uterino de aneurisma do $A D$ associado à hidropisia, em feto com traços sindrômicos.

\section{Relato do Caso}

Paciente de cor branca, 26 anos, Gesta II Para I, com 22 semanas de gestação, encaminhada ao serviço de medicina materno-fetal devido à derrame pericárdico, detectado em ecografia obstétrica de rotina. Ecocardiograma fetal afastou a presença de derrame pericárdico e aventou-se, como hipótese inicial, anomalia de Ebstein, com grande dilatação do AD e regurgitação tricúspide discreta. Após 2 semanas, o feto passou a apresentar sinais de hidropisia (ascite e edema de pele). Novo ecocardiograma permitiu avaliação mais detalhada da anatomia da valva tricúspide, não demonstrando implantação caudal valvar típica de Ebstein. 0 $A D$ aneurismático estendia-se cranialmente até a veia cava supe-

Correspondência: Silvio Henrique Barberato

Rua Saint Hilaire, 122/203 - 80240-140 - Curitiba, PR

E-mail: silviohb@cardiol.br

Recebido em 06/05/2004 - Aceito em 10/02/2005 rior e aorta ascendente e, caudalmente, para o ápice do coração, margeando o ventrículo direito (fig. 1). Foi discutido o uso de digital e diurético, porém a equipe assistente preferiu conduta expectante. Com 30 semanas de idade gestacional, ecografia de controle apontou retardo de crescimento intra-uterino e sinais de sofrimento fetal, optando-se pela interrupção da gravidez. Recémnascido (RN) de parto operatório, com peso de 2.250 gramas, APGAR 4 e 7 no primeiro e quinto minutos, respectivamente, havendo necessidade de ventilação mecânica. Ao exame físico, detectou-se edema generalizado, fácies sindrômico (formato triangular, testa proeminente, baixa implantação auricular) e hepatomegalia de consistência firme. O RN foi transferido para UTI neonatal, onde ecocardiograma transtorácico confirmou a doença (fig. 2). Não foram visibilizados contraste espontâneo ou presença de trombo no interior do aneurisma. Confirmou-se regurgitação tricúspide discreta e verificou-se a presença de comunicação interatrial pequena. O RN cursou com parada cardiorrespiratória e óbito com apenas 10 horas de vida, não tendo sido avaliado por geneticista. A necropsia mostrou aneurisma gigante de parede livre do AD com aspecto paper-thin, medindo $7 \times 2,8 \mathrm{~cm}$ e ocupando quase todo o mediastino. Valvas atrioventriculares e semilunares de aspecto macroscópico normal, ventrículos com dimensões preservadas e pericárdio sem alterações. Verificada comunicação interatrial do tipo forame oval com 1,6 mm de diâmetro. Fígado com tamanho aumentado, de consistência endurecida e aspecto nodular. Biópsia hepática revelou atresia de vias biliares. Não foi realizado cariótipo.

\section{Discussão}

Relatos de aneurisma do $A D$ têm sido feitos na infância ${ }^{5}$, idade adulta $^{3,4}$ e, muito raramente, na vida intra-uterina ${ }^{6,7}$. A maior revisão publicada sobre as malformações congênitas do $A D$, abrangendo 


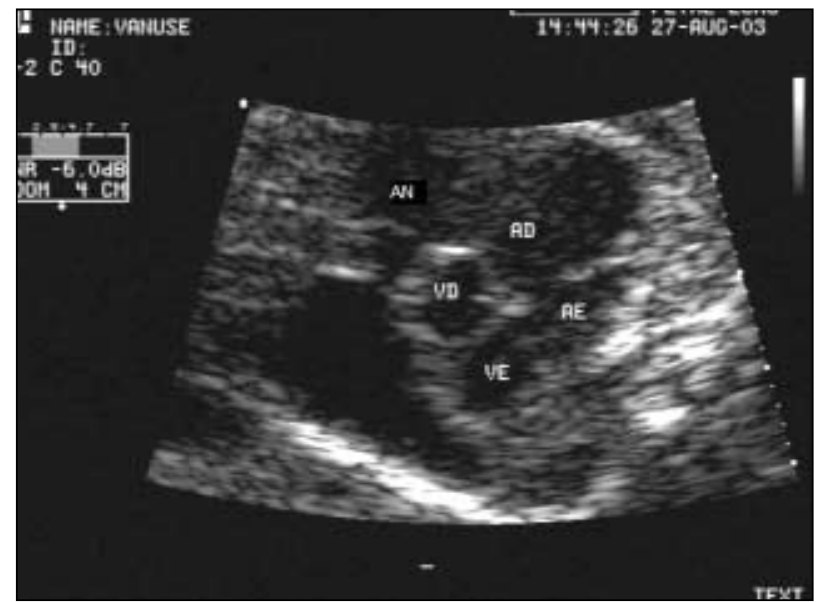

Fig. 1 - Ecocardiograma fetal: corte de 4 câmaras demonstrando aneurisma do átrio direito $(\mathrm{AN})$. $A D$ - átrio direito; $A E$ - átrio esquerdo; $V D$ - ventrículo direito; VE - ventrículo esquerdo.

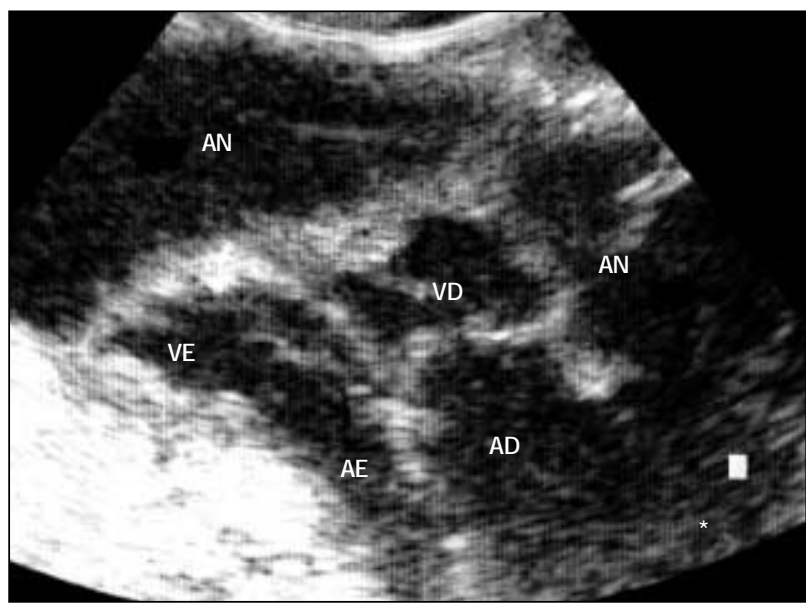

Fig. 2 - Ecocardiograma transtorácico: asteriscos assinalam o colo do aneurisma do átrio direito ( $A N)$. $A D$ - átrio direito; $A E$ - átrio esquerdo; VD - ventrículo direito; VE - ventrículo esquerdo. de 1955 a 19998, enumerou 60 casos de dilatação global congênita do AD entre 105 relatos, tornando-a o tipo mais comum de malformação desta câmara. Após minuciosa busca na literatura indexada, encontramos 4 casos de dilatação intra-uterina do $A D$ diagnosticada por meio de ultra-som fetal. Em apenas 2 deles houve o diagnóstico inicial correto de aneurisma do AD. No primeiro caso, após o achado de dilatação do $A D$ associada à insuficiência tricúspide, suspeitou-se de anomalia de Ebstein. Logo após o nascimento, ecocardiograma transtorácico firmou o diagnóstico correto ${ }^{1}$. No segundo caso, o aneurisma do AD foi diagnosticado em feto com 17 semanas de gestação, sem sinais de insuficiência cardíaca. Optou-se por interrupção da gestação e o estudo de necropsia foi confirmatório ${ }^{6}$. No terceiro caso, o diagnóstico de anomalia de Ebstein foi feito em feto de 35 semanas e confirmado após o nascimento. Aos 10 meses de vida, a criança apresentou sinais de insuficiência cardíaca direita refratária e foi submetida à cirurgia. No ato operatório, encontrou-se valva tricúspide normalmente posicionada e o AD teve sua parede livre parcialmente ressecada. A evolução foi assintomática por 4 anos de seguimento ${ }^{2}$. No quarto e último caso que seja do nosso conhecimento, o aneurisma do $A D$ foi precisamente diagnosticado em gestante de 32 semanas que passava por ecografia obstétrica de rotina e o período perinatal cursou sem intercorrências. A criança evoluiu assintomática até um ano de idade quando ecocardiogramas seriados demonstraram aumento progressivo do aneurisma (gerando compressão vascular) e aparecimento de trombo intracardíaco. Optou-se por tratamento cirúrgico que transcorreu sem complicações. Após acompanhamento por um ano, a criança permanecia saudável ${ }^{7}$.

0 presente caso, embora inicialmente também tenha sido confundido com anomalia de Ebstein, apresenta algumas peculiaridades. A grave evolução com hidropisia e sofrimento fetal, necessitando interromper a gravidez, não havia ainda sido relatada. Todos os casos previamente descritos foram achados de exame em eco- grafia obstétrica sistemática. Em nenhum deles o feto apresentava sinais de insuficiência cardíaca, a despeito de grande dilatação atrial. Na maioria dos casos, o diagnóstico do aneurisma do $A D$ só é feito em idade mais avançada, seja por achado de exame ${ }^{5}$, arritmias atriais ${ }^{4}$ e fenômenos embólicos ${ }^{9}$. Pode-se inferir que sua evolução no período fetal e na infância é comumente benigna e silenciosa. No presente caso, duas possibilidades podem ter levado à hidropisia: a provável síndrome que o feto portava (sem haver relação com o aneurisma em si) ou, alternativamente, devido à compressão de estruturas vasculares e ventrículo direito, obstruindo o retorno venoso e prejudicando o débito cardíaco. A grande dilatação do $A D$ e do anel tricúspide, causando pseudodeslocamento do folheto septal da valva, levou à impressão diagnóstica inicial errônea de anomalia de Ebstein. 0 fácies sindrômico associado à doença biliar intra-hepática e malformação cardíaca direita nos fez incluir no diagnóstico diferencial a síndrome de Alagille ${ }^{10}$. Entretanto, pelos critérios clássicos para definição da síndrome não podemos afirmar que se trata de um caso típico. Embora as malformações cardíacas direitas sejam as mais comuns nesta doença, não encontramos relato de aneurisma do $\mathrm{AD}$. Infelizmente, a não realização do cariótipo, conduta mandatória em casos de cardiopatia fetal grave com suspeita de síndrome, limitou o correto esclarecimento etiológico.

Nosso caso enfatiza o papel da ecocardiografia fetal no achado de cardiopatias congênitas graves e raras cujo diagnóstico não é realizado, o que pode acarretar mudanças nas taxas estatísticas e na terapêutica apropriada. Pela pouca experiência acumulada com o aneurisma do AD diagnosticado em vida intra-uterina, a conduta mais sensata parece ser a expectante. $\mathrm{Na}$ eventualidade do achado de dilatação atrial em exame ecográfico obstétrico, deve-se proceder ao ecocardiograma fetal com especialista habilitado para o diagnóstico diferencial do aneurisma do $A D$ com outras anomalias que cursam com aumento de $A D$, derrame pericárdico e tumores. 


\section{Referências}

1. Silva AM, Witsemburg M, Elzenza N, Stewart P. Idiopathic dilatation of the right atrium diagnosed in utero. Rev Port Cardiol 1992; 11: 161-3.

2. Reinhardt-Owlya L, Sekarski N, Hurni M, Laurini R, Payot M. Idiopathic dilatation of the right atrium simulating Ebstein's anomaly. Apropos of a case diagnosed in utero. Arch Mal Coeur Vaiss 1998; 91: 645-9.

3. Zeebregts CJ , Hensens AG, Lacquet LK. Asymptomatic right atrial aneurysm: fortuitous finding and resection. EurJ Cardiothorac Surg 1997; 11: 591-3.

4. Barberato SH, Barberato MF, Avila BM, Perretto S, Blume Ld Ldo R, Chamma Neto M. Aneurysm of the right atrial appendage. Arq Bras Cardiol 2002; 78: 236-41.

5. Chatrath R, Turek O, Quivers ES, Driscoll DJ , Edwards WD, Danielson GK. Asymptomatic giant right atrial aneurysm. Tex Heart Inst] 2001;28: 301-3.

6. Gross B, Petrikovsky B, Challenger M. Prenatal diagnosis of an aneurysm of the right atrium. Prenat Diagn 1996; 16: 1043-5.
7. Haut Cilly FB, Schleich J M, Lacour-Gayet F, Almange C. Right atrial compressive aneurysm with favorable outcome after surgery at the age of 1 month. Arch Mal CoeurVaiss 2002; 95: 487-90.

8. Binder TM, Rosenhek R, Frank H, Gwechenberger M, Maurer G, Baumgartner $H$. Congenital malformations of the right atrium and the coronary sinus: an analysis based on 103 cases reported in the literature and two additional cases. Chest 2000; 117: 1740-8.

9. Staubach P. Large right atrial aneurysm: rare cause of recurrent pulmonary embolism. ZKardiol 1998; 87: 894-9.

10. MCElhinney DB, Krantz ID, Bason $L$ et al. Analysis of cardiovascular phenotype and genotype-phenotype correlation in individuals with a J AG1 mutation and/or Alagille syndrome. Circulation 2002; 106: 2567-74. 